\title{
Direct visualization of common bile duct during laparoscopic treatment of choledocholithiasis
}

\author{
Bülent Kaya, Mehmet Mahir Fersahoğlu, Fatih Kılıç \\ Department of General Surgery, Fatih Sultan Mehmet Training and Research Hospital, İstanbul, Turkey
}

\begin{abstract}
Choledocolithiasis is an important pathology in hepatobiliary surgery. Minimally invasive methods are widely accepted in the management of choledocholithiasis. Laparoscopic and endoscopic approaches can be used. Presently described is the case of an 89-year-old male patient who was treated with laparoscopy and visualization of the common bile duct (CBD) was achieved using a 10-mm laparoscopic camera. Insertion of a laparoscopic camera into the CBD can be performed when the use of choledocoscopy or intraoperative cholangiography is impossible. A 5-mm laparoscopic camera may be even more useful for this purpose.
\end{abstract}

Keywords: Choledocolithiasis; common bile duct; laparoscopic camera.

\section{Introduction}

Choledocholithiasis is an important pathology in hepatobiliary surgery. It can cause many problems such as biliary colic, cholangitis. ${ }^{[1]}$ Proper treatment of choledocholithiasis is essential. After widespread use of endoscopic retrograde cholangiopancreaticography (ERCP), surgical approaches for choledocholithiasis decreased. In last years, laparoscopic surgical techniques were also improved for choledocholithiasis and widely accepted as a treatment modality. ${ }^{[2,3]}$

During laparoscopic management of choledocholithias, remnant stones are important problem. Intraoperative cholangiography and choledocoscopy are major tools for detecting common bile duct (CBD) stones in surgical operation. In this case report, we presented a patient who was treated with laparoscopy and visualisation of common bile duct was achieved by laparoscopic $10 \mathrm{~mm}$ camera.

\section{Case Report}

A 89 year old male admitted to emergency department with abdominal pain. His medical history was insignificant with only cholecystectomy. In physical examination, there was tenderness with palpation in right upper quadrant. The laboratory findings: bilirubin total: 2.57 (0.2-1.2 $\mathrm{mgr} / \mathrm{dl})$, bilirubin conjugated: $2.16(0.00-0.5 \mathrm{mgr} / \mathrm{dl})$, CRP: 22.8 (0-0.5 mgr/dl), amylase: 26 (25-125 U/L), white blood cell count: 10.4 (4.6-10.2 K/U). There was choledocholithiasis in abdominal computed tomography. The diameter of common bile duct was $20 \mathrm{~mm}$. The magnetic resonance cholangio pancreaticography was accordance with Computed tomography. There was no stone in right or left hepatic duct. The ERCP was performed. It was 
unsuccessful due to large size of the stone. Patient was explorated with laparoscopy. The abdominal trochars were inserted as in standart laparoscopic cholecystectomy. There was no need for additional trochar insertion during operation. Common bile duct was explorated. The diameter of common bile duct was approximately 2.5-3 $\mathrm{cm}$. CBD was dissected. It was opened with a vertical $3 \mathrm{~cm}$ incision. There was a stone about $2 \mathrm{~cm}$ in diameter in common bile duct. There was also biliary sludge. The stone was extracted. Common bile duct was irrigated with $50 \mathrm{cc}, 0.9 \% \mathrm{NaCl}$ solution. Due to large size of CBD, proximal and distal part of the duct were examined

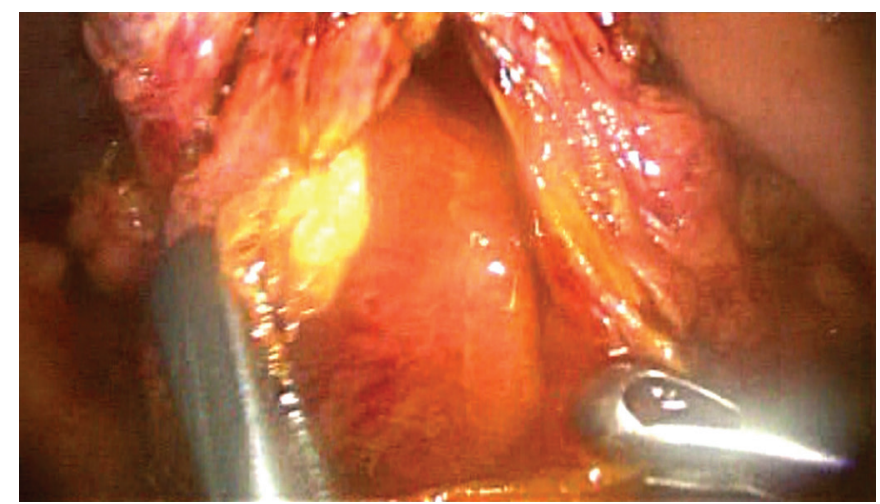

Figure 1. Appreance of proximal common bile duct after stone extraction.

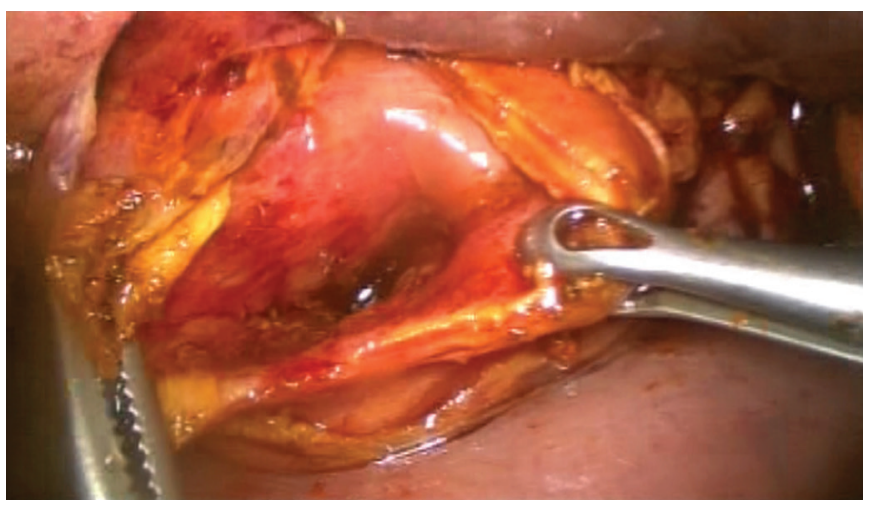

Figure 2. Distal common bile duct with no stone.

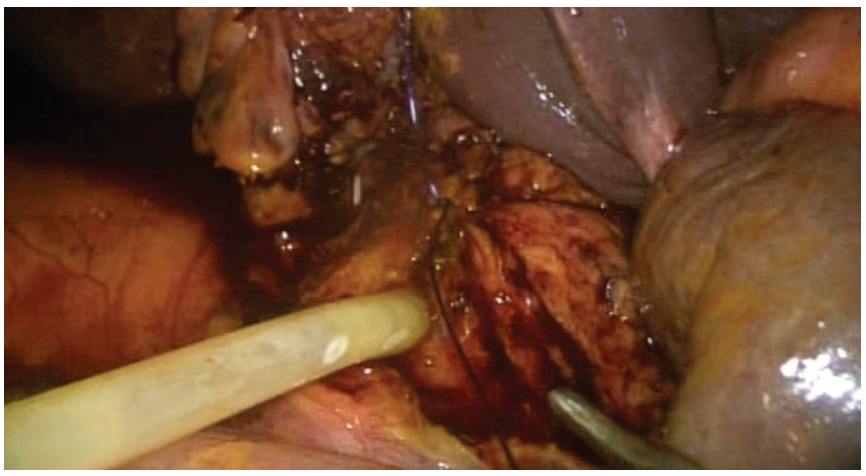

Figure 3. Closure of common bile duct with t-tube. easily with laparoscopic camera (Figures 1, 2). There was no retained stone. Cholangiography could not be performed due to technical problems. The common bile duct was closed with 3/0 PDS suture after insertion of T-tube (Figure 3). The postoperative period was uneventful. The patient was discharged from hospital in 7. postoperative day. The T-tube was withdrawn in 21. postoperative day without complication.

\section{Discussion}

Choledocholithiasis is detected approximately 10\%-20\% of patients with gallbladder stones and its incidence increases with advancing age. It was stated that at least 3\%$10 \%$ of patients operated due to cholelithiasis will have CBD stones. ${ }^{[4]}$

Minimal invasive methods in management of choledocholithiasis is widely accepted (Table 1). Laparoscopic and endoscopic approaches for choledocholithiasis can be effective treatment choice and was first described in 1991 by Petelin. ${ }^{[5]}$ Like other laparoscopic interventions, significant expertise and experience is required to achieve high success rates. Quaresima S et. al operated 46 patients due to cholelithiasis and choledocholithiasis with laparoscopic approach. ${ }^{[6]}$ They performed transverse choledochotomy and T-tube drainage. Among all patients with 17 year follow up, ductal stone recurrence occurred in one case (2.1\%) and managed by ERCP with endoscopic sphincterotomy. There were no patients with signs of bile stasis and no patient showed any imaging evidence of CBD stricture at the site of choledochotomy. They concluded that laparoscopic transverse choledochotomy during laparoscopic cholecystectomy is safe and effective approach, with no evidence of common bile duct stricture. It was also suggested that choledochotomy must be performed with a correct surgical technique to avoid ischemia with a T-tube of small diameter. T-tube should left in place for enough time maturating sinus tract.

Table 1. Management of gallbladder and common bile duct stones with minimal invasive techniques

\begin{tabular}{ll} 
Single step approach & Two step approach \\
\hline $\begin{array}{l}\text { LC+LCBDE } \\
\text { or } \\
\text { LC+Intraoperative ERCP }\end{array}$ & Preoperative ERCP+LC \\
\hline
\end{tabular}

LCBDE: Laparoscopic common bile duct exploration; LC: Laparoscopic cholecystectomy. 
The visulisation of common bile duct is important in management of choledocholithiasis. Retained stones can be important problem. Intraoperative cholangiography, which was first introduced by Mirizzi in 1931 is beneficial for delineating biliary anatomy and identifying stones in the CBD. But it has also some disadvantages. It results in the lengthtening of the operation (mean time of $30 \mathrm{~min}$ utes) and increase in costs of surgical operation. It has also associated with false positive results in $1-3 \%$ of cases, resulting in unnecessary biliary tract interventions. ${ }^{[7]}$

Flexable choledochoscope is one of the most important tool that can be used for visualisation of common bile duct. It can be introduced intraoperatively through the cystic duct or directly into the common bile duct. Stone extraction with the choledochoscope can be successful in $75 \%$ to $95 \%$ of the cases. ${ }^{[8]}$ So it may be the best and easy method for common bile duct visualization.

In this case report, we used the laparoscopic $10 \mathrm{~mm}$ camera as a choledochoscope. It can be inserted directly into the common bile duct specially in patients with a common bile duct diameter above $2-3 \mathrm{~cm}$ and can be beneficial in cases that flexable choledocoscope is not found. A laparoscopic $5 \mathrm{~mm}$ camera may be even more easily inserted into the common bile duct. Main advantage of using laparoscopic camera is it's facility. On the other hand, camera can see only bifurcation of CBD. Any stone in right or left hepatic duct can be missed. The visualisation of the distal part of the CBD is also important. It should be clearly seen in exploration. To avoid remnant stones after laparoscopic CBD exploration, preoperative evaluation of biliary anatomy with radiological tools are mandatory.
In conclusion, surgical technique that explained in this case report is not gold standart, it can be used as an alternative technique in CBD exploration.

\section{Disclosures}

Peer-review: Externally peer-reviewed.

Conflict of Interest: None declared.

\section{References}

1. Desai R, Shokouhi BN. Common bile duct stones - their presentation, diagnosis and management. Indian J Surg 2009;71:229-37. [CrossRef]

2. Berthou JCh, Dron B, Charbonneau P, Moussalier K, Pellissier L. Evaluation of laparoscopic treatment of common bile duct stones in a prospective series of 505 patients: indications and results. Surg Endosc 2007;21:1970-4. [CrossRef]

3. Taylor CJ, Kong J, Ghusn M, White S, Crampton N, Layani L. Laparoscopic bile duct exploration: results of 160 consecutive cases with 2-year follow up. ANZ J Surg 2007;77:440-5.

4. Schirmer BD, Winters KL, Edlich RF. Cholelithiasis and cholecystitis. J Long Term Eff Med Implants 2005;15:329-38.

5. Petelin JB. Laparoscopic common bile duct exploration. Surg Endosc 2003;17:1705-15. [CrossRef]

6. Quaresima S, Balla A, Guerrieri M, Lezoche G, Campagnacci R, D'Ambrosio G, et al. Results of Medium Seventeen Years' Follow-Up after Laparoscopic Choledochotomy for Ductal Stones. Gastroenterol Res Pract 2016;2016:9506406.

7. Iorga C, Cirimbei S, Strambu V, Popa F. Intraoperative cholangiography still a current investigation. J Med Life 2013;6:399-402.

8. Wood T, Macfadyen BV Jr. Diagnostic and therapeutic choledochoscopy. Semin Laparosc Surg 2000;7:288-94. [CrossRef] 